\title{
Current Algebra Representation for the 3+1 Dimensional Dirac-Yang-Mills Theory
}

\author{
Jouko Mickelsson *
}

Fakultät für Physik der Universität Freiburg, Hermann-Herder-Strasse 3,

D-7800 Freiburg, Federal Republic of Germany

\begin{abstract}
The structure of the current algebra representation in the state space of fermions in an external Yang-Mills field in $3+1$ space-time dimensions is analyzed; the topology of the vector space is determined by a countable family of semi-definite inner products. We show that there is no hermitian non-trivial Hilbert space representation such that the energy is bounded from below. The structure of the Hilbert space for the quantized coupled Dirac-Yang-Mills system is discussed and the existence of the vacuum vector and the cancellation of commutator anomalies is described in terms of complex line bundles over infinite-dimensional Grassmannians.
\end{abstract}

\section{Introduction and Notation}

We shall study the problem of quantizing a coupled Dirac-Yang-Mills system in the case when the dimension of space-time is $3+1$ and the gauge group $G$ is nonabelian and compact. In particular, we want to 1) determine the structure of the relevant current algebra representation, 2) discuss the anomaly cancellation in the Hilbert space of the quantized coupled system using previous results on the structure of complex line bundles over infinite-dimensional Grassmannians [MR].

Let $M$ be a compact oriented spin manifold of dimension 3 and $S$ a tensor product of the Dirac spinor bundle over $M$ (fiber $\mathbb{C}^{2} \oplus \mathbb{C}^{2}$ ) with a topologically trivial vector bundle, the structure group of the latter being $G$. Let $L_{2}(S)$ denote the space of square-integrable sections of $S$ with respect to a fixed fiber metric of $S$ and a measure on $M$. Let $D_{A}$ denote a covariant Dirac operator in $S$ defined by a vector potential $A$ taking values in the Lie algebra of $G$. Locally we can write

$$
D_{A}=\sum_{k=1}^{3} \alpha^{k}\left(\nabla_{k}+A_{k}\right)+\gamma_{0} m
$$

\footnotetext{
* Permanent address: Department of Mathematics, University of Jyväskylä, Seminaarinkatu 15, SF-40100 Jyväskylä, Finland
} 
where $\alpha_{k}=\gamma_{0} \gamma_{k}$ and the $\gamma_{\mu}$ 's are the Dirac matrices; $\nabla_{k}$ is the covariant derivative (determined by the spin connection form) in the $k^{\text {th }}$ coordinate direction. We define a splitting $L_{2}(S)=H_{+}(A) \oplus H_{-}(A)$, such that $H_{+}(A)$ consists of linear combinations of eigenvectors of $D_{A}$ corresponding to non-negative eigenvalues and $H_{-}(A)$ is the orthogonal complement of $H_{+}(A)$. Let $M G$ be the group (under point-wise multiplication) of smooth maps $M \mapsto G$. We have a natural action of the group of gauge transformations $M G$ in $L_{2}(S)$ defined point-wise, $(g \cdot \psi)(x)=g(x) \cdot \psi(x)$. With respect to the splitting defined above, we can write the linear operator in $L_{2}(S)$ defined by the gauge transformation $g$ in the block form

$$
g=\left(\begin{array}{ll}
a & b \\
c & d
\end{array}\right)
$$

where $a: H_{+} \rightarrow H_{+}, b: H_{-} \rightarrow H_{+}, c: H_{+} \rightarrow H_{-}, d: H_{-} \rightarrow H_{-}$are continuous linear operators. Denote by $I_{p}$ the Schatten ideal of linear operators $T$ satisfying $\operatorname{tr}|T|^{p}<\infty$. It is known that the blocks $b, c$ of $g$ belong to $I_{4}$ and $a, d$ are Fredholm operators, [C, PS]. We denote by $G L_{2}$ the group of all invertible linear operators of this type. Thus we have a homomorphism $M G \rightarrow G L_{2}$, and it follows that a representation of $G L_{2}$ gives automatically a representation of the group of gauge transformations.

In $1+1$ dimensional field theory the representation of the current algebra in the Fock space of the second quantized Dirac field is not a true representation but a projective one. It is a true representation of a central extension of the current algebra. The central extension is precisely an affine Kac-Moody algebra when $G$ is a compact simple group. In the $3+1$ dimensional case the situation is worse than that. One has to define an extension of the current algebra by an infinitedimensional abelian ideal ("operator valued Schwinger terms"), [M1, F, S]. (This type of Schwinger terms were derived perturbatively in the case of an abelian vector potential in $[\mathrm{J}]$.) For this reason one has to go from the Fock space to a bigger space; the Fock space is simply not invariant under gauge transformations. In [MR] a study of the representations of the appropriate extension $\widehat{G L} L_{2}$ of $G L_{2}$ was initiated. A linear representation of $\widehat{G L}_{2}$ was constructed in the space of sections of a complex line bundle DET* over an infinite-dimensional Grassmannian $\mathrm{Gr}_{2}$. The space contains the Fock space (= the space of holomorphic sections) as a (non-invariant) subspace. However, the question whether there is an invariant inner product in the representation space was left open.

This paper grew out from an effort to construct an invariant inner product in the space $\Gamma$ of sections of $\mathrm{DET}_{2}^{*}$. In that respect the result is negative: We prove that there does not exist any non-trivial hermitian representation of the Lie algebra of $\widehat{G L}_{2}$ such that the energy is bounded below (Proposition 3.21). The best we can do is to define the topology of the space of sections with a countable family of semi-definite inner products. We analyze the structure of the space $\Gamma$ and we show how it decomposes to "highest weight" representations of the infinitedimensional general linear Lie algebra $\mathrm{gl}(\infty)$. These are not highest weight representations in the standard terminology since we are using a "triangular splitting" of the Lie algebra which differs from the standard splitting to upper triangular, diagonal and lower triangular matrices, hence the quotation marks. 
We need some notions and results from [MR]. The Grassmannian $\mathrm{Gr}_{2}$ is defined as the homogeneous space $G L_{2} / N$, where $N$ is the group of operators $g$ with $c=0$. The points on the Grassmannian are infinite-dimensional planes $W=g \cdot H_{+}$with the properties i) the orthogonal projection $W \rightarrow H_{+}$is a Fredholm operator, and ii) the projection $W \rightarrow H_{-}$is in $I_{4}$. A Grassmannian plane $W$ is uniquely determined by a unitary hermitian operator $F$ such that $\left.F\right|_{W}=1$ and $\left.F\right|_{W^{\perp}}=-1$. When written in the block form $F=\left(\begin{array}{ll}F_{11} & F_{12} \\ F_{21} & F_{22}\end{array}\right)$ the operators $F_{12}, F_{21} \in I_{4}$ and $F_{11}-1, F_{22}+1 \in I_{2}$. Fix an orthonormal basis $e=\left\{e_{i}\right\}_{i \in \mathbb{N}}$ of $H_{+}$. The Stiefel manifold $\mathrm{St}_{2}$ consists of all admissible basis of all Grassmann planes: a basis $w=\left\{w_{i}\right\}_{i \in \mathbb{N}}$ of $W$ is admissible if the matrix $w_{+}$defined by

$$
p r_{H+} w_{i}=\sum_{j \in \mathbb{N}}\left(w_{+}\right)_{j i} e_{j}
$$

is of the type $1+k$ with $k \in I_{2}$. We denote by $G L^{2}$ the group of all invertible operators in $H_{+}$of the form $1+k, k \in I_{2}$. The determinant bundle $\mathrm{DET}_{2}$ is $\mathrm{St}_{2} \times \mathbb{C} / G L^{2}$, where the action of $G L^{2}$ is defined by

$$
(w, \lambda) \cdot t=\left(w t, \lambda \omega_{2}\left(w_{+}, t\right)\right) .
$$

Here $\omega_{2}$ is a cocycle for the right action of $G L^{2}$ on $\mathrm{St}_{2}$ given by the formula $\omega_{2}\left(w_{+}, t\right)=\operatorname{det}_{2} t \cdot \exp \left(-\operatorname{tr}\left(w_{+}-1\right)(t-1)\right)$, where $\operatorname{det}_{2} t$ is the renormalized determinant which can be defined as the limit $n \rightarrow \infty$ of $\operatorname{det} t_{n} \cdot \exp \left(-\operatorname{tr}\left(t_{n}-1\right)\right)$ for any sequence $\left\{t_{n}\right\}$ of operators in $1+I_{1}$ such that $t_{n} \rightarrow t$ in the $I_{2}$ topology. The action of $t$ on $w$ is defined by $w_{i}^{\prime}=\sum w_{j} t_{j i}$, the matrix elements $t_{j i}$ being defined with respect to the basis $e$.

The action of $G L_{2}$ on $\mathrm{Gr}_{2}$ cannot be lifted to a (projective) action on the total space of the bundle $\mathrm{DET}_{2}$. Instead, one has to define an extension $\widehat{G L}_{2}$ of $G L_{2}$ by an infinite-dimensional abelian normal subgroup $A_{2}$ which acts on DET 2 . The group $A_{2}$ consists of all functions $\exp (\eta+\operatorname{tr} \xi(F-\varepsilon))$ on $\mathrm{Gr}_{2}$, where $\eta \in \mathbb{C}$ and $\xi=\left(\begin{array}{ll}\xi_{11} & \xi_{12} \\ \xi_{21} & \xi_{22}\end{array}\right)$ is any linear operator in $H_{+} \oplus H_{-}$such that $\xi_{11}, \xi_{22} \in I_{2}$ and $\xi_{21}, \xi_{12} \in I_{4 / 3}$. The action of an element $f$ of $A_{2}$ in the fiber of DET ${ }_{2}$ over $W$ is given by multiplication by $f(W)$. As a vector space the Lie algebra $\hat{g} l_{2}$ of $\widehat{G L} L_{2}$ is the sum of the Lie algebra $\mathrm{gl}_{2}$ with the abelian Lie algebra $a_{2}$; the latter is the sum of $\mathbb{C}$ with the vector space consisting of operators of the form $\xi$ above. The commutators are

$$
[(X, f),(Y, h)]=\left([X, Y], X \cdot h-Y \cdot f+c_{2}(X, Y ; \cdot)\right),
$$

where the commutator $[X, Y]$ is the usual operator commutator, $X \cdot f$ denotes the Lie derivative of the function $f$ to the direction of the vector field $X$ (generated by the natural action of $G L_{2}$ on $\mathrm{Gr}_{2}$ ) and $c_{2}$ is a two-cocycle defined by

$$
c_{2}(X, Y ; F)=\frac{1}{8} \operatorname{tr}[[X, \varepsilon],[Y, \varepsilon]](F-\varepsilon) .
$$

If $f(W)=\eta+\operatorname{tr} \xi(F-\varepsilon)$, then $(X \cdot f)(W)=\eta^{\prime}+\operatorname{tr} \xi^{\prime}(F-\varepsilon)$ with $\eta^{\prime}=\operatorname{tr}[X, \xi] \varepsilon$ and $\xi^{\prime}=[X, \xi]$. Since $\widehat{G L}_{2}$ acts on $\mathrm{DET}_{2}$, it acts naturally on the space of sections of the dual bundle DET 2 . The important difference when compared to the $1+1$ dimensional case is that the space of holomorphic sections is not invariant under 
the group $\widehat{G L}_{2}$. In the $1+1$ dimensional case the space of holomorphic square integrable sections can be identified as the Fock space of the second quantized Dirac field [PS, Pi]. In the $3+1$ dimensional case the representation of the current algebra cannot be defined in the Fock space. The vacuum vector is the holomorphic section $\psi(w)=\operatorname{det}_{2} w_{+}$, but a section obtained from $\psi_{0}$ by the group action is in general not holomorphic. Any section can be thought of as a equivariant function on $\mathrm{St}_{2}$ satisfying $\psi(w t)=\psi(w) \omega\left(w_{+}, t\right)$ for $t \in G L^{2}$.

In Sect. 2 we shall discuss some aspects of the representation theory of finitedimensional linear groups and their extensions by abelian ideals using the Mackey theory of induced representations. The results will be used in Sect. 3, where we shall study the decomposition of the representation of $\hat{\mathrm{g}} l_{2}$ in $\Gamma$ into highest weight representations of the subalgebra $\hat{\mathrm{g}} \mathrm{l}(\infty)$ of $\hat{\mathrm{g}} l_{2}$.

In Sect. 4 we discuss the structure of the Hilbert space for the fully quantized Dirac-Yang-Mills system. The space consists of sections of a vector bundle $B$ over the space $\mathscr{A}$ of smooth vector potentials in the 3 dimensional physical space. To each vector potential $A$ one associates the fermionic vector space $B_{W}$ consisting of sections of the line bundle DET* defined using the Dirac operator $D_{A}$. The space $\mathscr{A}$ can be embedded into the Grassmannian $\mathrm{Gr}_{2}$ and the bundle $B$ can be extended to a vector bundle over $\mathrm{Gr}_{2}$. The vacuum sector $\mathrm{Vac}$ of $B$ is twisted and there is no continuous choice of the vacuum $\psi_{W} \in B_{W}$ for all $W \in \mathrm{Gr}_{2}$. One has to introduce a ghost field. Mathematically this means that one has to tensor the bundle $B$ with the line bundle $\mathrm{DET}_{2}$. The new bundle $\bar{B}$ has a continuously defined vacuum at each point $W \in \mathrm{Gr}_{2}$. Furthermore, the vacuum is gauge invariant (invariant under the group $G L_{2}$ ) and the commutator anomalies for $\mathrm{gl}_{2}$ arising from the action in the

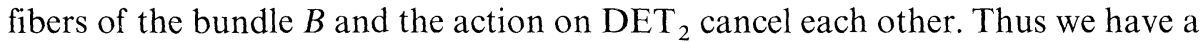
true representation of $G L_{2}$ on sections of $\bar{B}$. The Fock space $\mathscr{F}_{W} \subset B_{W}$ at $W \in \mathrm{Gr}_{2}$ consists of holomorphic sections of DET 2 . The Fock bundle $\mathscr{F}$ over $\mathrm{Gr}_{2}$ is not invariant under the action of $\widehat{G L}_{2}$ but the modified Fock bundle $\overline{\mathscr{F}}=\mathscr{F} \otimes \mathrm{DET}_{2}$ is invariant and $\mathrm{Vac}$ is a holomorphic subbundle of $\overline{\mathscr{F}}$. The holomorphic structures in line bundles over Grassmannians appear here in a similar way as in the cancellation of diffeomorphism anomalies in string theory, [BR, M2].

\section{Some Facts about Representations of Finite-Dimensional Linear Groups}

Let $G L(N)$ denote the group of invertible $N \times N$ complex matrices and $U(N)$ the subgroup of unitary matrices. Denote by $e_{i j}$ the matrix with all entries $=0$ except the element at the position $(i, j)$ which is equal to one. We shall denote Lie algebras by lower case boldface letters. A Cartan subalgebra $\mathbf{h}$ of $\mathrm{gl}(N)$ is spanned by the elements $e_{i i}$. We shall consider especially the groups $G L(2 N)$, and for reasons which shall become clear in the next section we shall index a basis in $\mathbb{C}^{2 N}$ by non-zero integers in the range $-N \leqq i \leqq N$. An irreducible finite-dimensional representation of $G L(2 N)$ is characterized (up to an equivalence) by the highest weight $\lambda=\left(\lambda_{1}, \lambda_{2}, \ldots, \lambda_{N}, \lambda_{-N}, \lambda_{-N+1}, \ldots, \lambda_{-1}\right)$, where the $\lambda_{i}$ 's are all integers and form a non-increasing sequence from left to right. Let $\mathbf{k}_{+}$be the subalgebra of $\operatorname{gl}(2 N)$ spanned by the generators

$$
e_{i j}, e_{-j,-i} \text { with } 0<i<j \leqq N ; \quad e_{i,-j} \text { with } i, j>0
$$


and let $\mathbf{k}_{\text {- }}$ be the subalgebra spanned by

$$
e_{j i}, e_{-i,-j} \text { with } 0<i<j \leqq N ; \quad e_{-i, j} \text { with } i, j>0 .
$$

Let $V(\lambda)$ denote the representation space. There is a unique (up to a complex factor) highest weight vector $x(\lambda)$ such that

$$
k_{+} x(\lambda)=0, \quad e_{i i} x(\lambda)=\lambda_{i} x(\lambda) .
$$

The choice of the splitting $\mathrm{gl}(2 N)=\mathbf{k}_{+} \oplus \mathbf{h} \oplus \mathbf{k}_{-}$may appear a little strange, but the motivation is clarified in the next section.

An irreducible representation $\lambda$ of $G L(2 N)$ decomposes to a direct sum of irreducible representations of the subgroup $G L(2 N-1)$ consisting of matrices with $A_{11}=1, A_{1 i}=A_{i 1}=0$ for $i \neq 1$. The highest weights $\lambda^{2 N-1}$ of $\operatorname{gl}(2 N-1)$ which occur in the reduction are those which satisfy the inequalities $\lambda_{1} \geqq \lambda_{1}^{2 N-1} \geqq \lambda_{2}$ $\geqq \lambda_{2}^{2 N-1} \geqq \ldots \geqq \lambda_{-2} \geqq \lambda_{-2}^{2 N-1} \geqq \lambda_{-1}$; each of these representations $\lambda^{2 N-1}$ occur with multiplicity $=1$ in the reduction. Thus with the help of the subgroup chain $G L(1) \subset G L(2) \subset \ldots \subset G L(2 N)$ one can label a basis in the representation space $V(\lambda)$. A Gelfand-Zetlin pattern $\left(\lambda_{i}^{j}\right)$ consists of rows of integers $\lambda_{i}^{j}$, where the row index $1 \leqq j \leqq 2 N$ refers to the subgroups $G L(j)$, each row being a highest weight of an irreducible representation of the corresponding subgroup, the top row is $\lambda_{i}^{2 N}=\lambda_{i}$, and the allowed values in the $j^{\text {th }}$ row are obtained by inequalities similar to those above from the values the $\lambda$ 's in the $(j+1)^{\text {th }}$ row (see Fig. 1); each $\lambda_{i}^{j}$ is between the two $\lambda$ 's immediately above. To each Gelfand-Zetlin pattern there corresponds a vector in a basis of $V(\lambda)$. One can define an inner product in $V(\lambda)$ such that the basis labelled by the GZ patterns is orthonormal. The representation of the subgroup $U(2 N)$ is unitary with respect to this inner product. Furthermore, the restriction to $U(2 N)$ is irreducible.

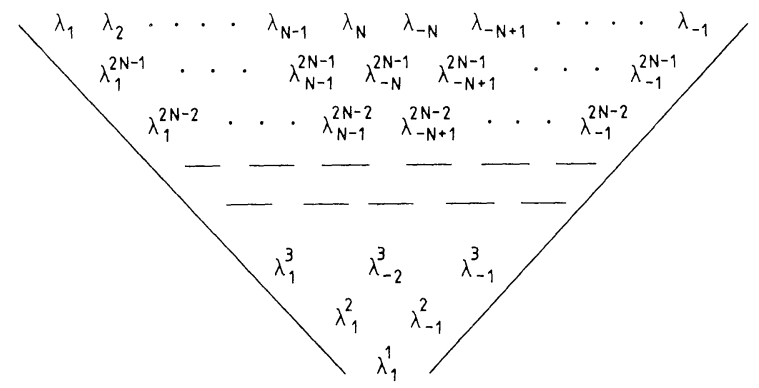

Fig. 1. Gelfand-Zetlin pattern for the reduction $U(2 N) \supset U(2 N-1) \supset \ldots \supset U(1)$

We need also some information about the reduction of a representation $\lambda$ of $G L(2 N)$ to the subgroup $G L(N)_{+} \times G L(N)_{-}$, where the first subgroup transforms only the components of a vector in $\mathbb{C}^{2 N}$ with positive indices and the second with negative indices. Suppose first that $\lambda_{-1} \geqq 0$. (In this case $\lambda_{i} \geqq 0 \forall i$.) There is a graphical rule which tells us which of the representations $\mu \times v$ of $G L(N)_{+}$

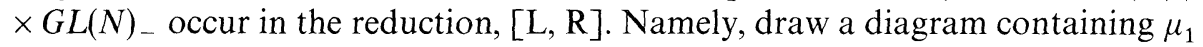
boxes in the first (top) row, $\mu_{2}$ boxes in the second row, .., $\mu_{N}$ boxes in the last (bottom) row (see Fig. 2). Add to the ends of the rows first $v_{-N}$ boxes labelled by 1 , 


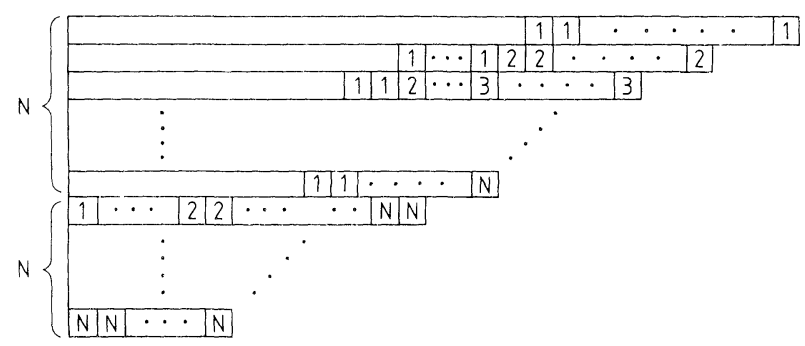

Fig. 2. Pattern for computing the multiplicities $m_{\mu v}^{\lambda}$

then $v_{-N+1}$ boxes labelled by $2, \ldots$, finally $v_{-1}$ boxes labelled by $\mathrm{N}$, where $v_{-N}$ $\geqq v_{-N+1} \geqq \ldots \geqq v_{-1}, \mu_{1} \geqq \mu_{2} \geqq \ldots \geqq \mu_{N}$, with the following restrictions: A) No label should occur twice in the same column, B) At each step the lengths of the rows in the diagram should form a non-increasing sequence, from top to bottom, C) When reading from the right to the left and from the top to the bottom, at each step the number of 1 's should be $\geqq$ the number of 2 's $\geqq \ldots \geqq$ the number of N's. The number of different ways how the box diagram with the row lengths $\left(\lambda_{1}, \lambda_{2}, \ldots, \lambda_{-1}\right)$ can be obtained from the data $(\mu, v)$ is equal to the multiplicity $m_{\mu \nu}^{\lambda}$ of the representation $\mu \times v$ of $G L(N)_{+} \times G L(N)_{-}$in $\lambda$. Obviously a necessary condition for $m_{\mu \nu}^{\lambda} \neq 0$ is $\sum \lambda_{i}=\sum \mu_{i}+\sum v_{i}$. The general case (when some of the $\lambda_{i}$ 's may be negative) is obtained by noting that the multiplicity of $\mu \times v$ in $\lambda$ is equal to the multiplicity of $\mu^{\prime} \times v^{\prime}$ in $\lambda^{\prime}$, where $\mu_{i}^{\prime}=\mu_{i}-\lambda_{N}, v_{i}^{\prime}=v_{i}-\lambda_{N}, \lambda_{i}^{\prime}=\lambda_{i}-\lambda_{N}$.

We shall need the multiplicities when $\mu=(1,1, \ldots, 1)$ and $v=(0,0, \ldots, 0)$. Using the graphical rules one arrives at the following result:

Lemma 2.1. $m_{\mu \nu}^{\lambda}=0$ or $=1$ and the non-zero values occur when $\lambda_{i}+\lambda_{-i}=1$ for $1 \leqq i \leqq N$.

Let $\operatorname{Gr}(N)$ be the Grassmannian of $N$-dimensional complex planes in the space $\mathbb{C}^{2 N}$. Define the maps $f_{i j}: \operatorname{Gr}(N) \rightarrow \mathbb{C}$ by $f_{i j}(F)=\operatorname{tr} e_{i j} F$, where we have parametrized the $N$-planes $W$ in $\mathbb{C}^{2 N}$ by unitary hermitian $2 N \times 2 N$ matrices $F$ such that $F z=z$ for $z \in W$ and $F z=-z$ for $z \in W^{\perp}$. The group $U(2 N)$ acts on the functions $f: \operatorname{Gr}(N) \rightarrow \mathbb{C}$ by $(g \cdot f)(F)=f\left(g^{-1} F g\right)$. In particular,

$$
g \cdot f_{i j}=\sum_{k, l} g_{k i} \bar{g}_{l j} f_{k l} \text {. }
$$

We define $\hat{U}(2 N)$ as the semidirect product of $U(N)$ and the abelian group $U A(N)$ consisting of maps $\exp \left(\eta+\sum \xi_{i j} f_{i j}\right)$, where $\eta \in i \mathbb{R},\left(\xi_{i j}\right)$ is an antihermitian $2 N \times 2 N$ matrix and the multiplication in $U A(N)$ is defined pointwise. The complexification $\widehat{G L}(2 N)=G L(2 N) \ltimes A(N)$ is defined by letting $\eta, \xi$ be arbitrary.

For each complex $2 N \times 2 N$ matrix $\sigma$ we define a one-dimensional representation $\varrho_{\sigma}$ of $A(N)$ by $\varrho_{\sigma}\left(\exp \left(\eta+\sum \xi_{i j} f_{i j}\right)\right)=\exp (\eta+\operatorname{tr} \sigma \xi)$. The group $G L(2 N)$ acts on the parameter $\sigma$ in the dual of $A(N)$ through $\sigma \mapsto g^{-1} \sigma g$. The stability subgroup of $G L(2 N)$ at the point $\sigma=i \varepsilon$,

$$
\varepsilon=\operatorname{diag}(1,1, \ldots, 1,-1,-1, \ldots,-1),
$$

(N times 1 and $\mathrm{N}$ times -1 ) is $G L(N)_{+} \times G L(N)_{-}$. We fix the representation $\mu \times v$ of the stability group by setting $\mu_{i}=1$ and $v=0$. Let $D$ denote the representation 
$\left(g_{+}, g_{-}, h\right) \mapsto \operatorname{det} g_{+} \cdot \varrho_{i \varepsilon}(h)$ of the semidirect product $\left(G L(N)_{+} \times G L(N)_{-}\right) \triangleright A(N)$ and let $T$ be the representation of $\widehat{G L}(2 N)$ induced by the representation $D$. According to the Mackey theory the representation $T$ is irreducible. From the Mackey subgroup theorem it follows that an irreducible representation $\lambda$ of $G L(2 N)$ occurs in the representation $T$ with equal multiplicity $m_{\mu \nu}^{\lambda}$ as the representation $\mu \times v$ of $G L(N)_{+} \times G L(N)_{-}$occurs in the reduction of the representation $\lambda$ to the subgroup $G L(N)_{+} \times G L(N)_{\ldots}$.

The restriction of $T$ to the subgroup $U(2 N) \triangleright U A(N)$ is unitarizible. The Hilbert space $V$ which carries the representation $T$ consists of the squareintegrable sections of the dual determinant bundle DET* over the Grassmannian $\operatorname{Gr}(N)$. The sections of DET* are by definition functions $\psi$ on $G L(2 N)$ which satisfy the condition

$$
\psi(g t)=\psi(g) \operatorname{det} a \text { for } t=\left(\begin{array}{ll}
a & b \\
0 & d
\end{array}\right),
$$

where $a, b, d$ are $N \times N$ matrices. Equivalently, a section is a function $\psi: U(2 N) \rightarrow \mathbb{C}$ such that the above relation holds for all unitary matrices $g, t$. The inner product of two sections $\psi, \psi^{\prime}$ is given by

$$
\left\langle\psi, \psi^{\prime}\right\rangle=\int_{\operatorname{Gr}(N)} \psi \bar{\psi}^{\prime} d m
$$

where $d m$ is the $U(2 N)$ invariant measure on $\operatorname{Gr}(N)$. The vacuum vector is the section $\psi_{0}(g)=\operatorname{det} a$, where $g=\left(\begin{array}{ll}a & b \\ c & d\end{array}\right)$. In the reduction of the representation $T$ with respect to the subgroup $U(N)_{+} \times U(N)_{-}$this is the vector (unique up to a constant) which transforms according to the one-dimensional representation $\mu \times v$. Since the representation $T$ is irreducible, a complete basis in $V$ can be obtained by applying the elements of the enveloping algebra $\mathscr{U}(\operatorname{gl}(2 N))$ to the vacuum. We shall now describe in detail how an orthonormal basis is constructed. In fact, it is sufficient to construct an orthonormal basis in the subspace $V_{+}=\left\{x \in V \mid \mathbf{k}_{+} x=0\right\}$ of highest weight vectors for the subalgebra gl $(2 N)$. Namely, one can apply the known methods (lowering operator technique, for example) from the theory of finite-dimensional representations of unitary groups to obtain a basis inside of each representation of $\mathrm{gl}(2 N)$ labelled by the highest weight $\lambda$. To obtain a basis in $V_{+}$we apply the theory of step algebras (which is a generalization of the lowering operator technique [NM]), [M3, H, Z]. For each pair of indices $-N \leqq i, j \leqq N$ there is an element $s_{i j}$ in the enveloping algebra of $\operatorname{gl}(2 N)$ such that

$$
\left[e_{k k}, s_{i j}\right]=\left(\delta_{k i}-\delta_{k j}\right) s_{i j}, z s_{i j} \in \mathscr{U}(\mathrm{gl}(2 N)) \mathbf{k}_{+} \forall z \in \mathbf{k}_{+} .
$$

From the second relation it follows that $V_{+}$is invariant under the action of the elements $s_{i j}$. From the general theory of step algebras follows that any element of $V_{+}$can be written as a polynomial in the elements $s_{i j}$ acting on the vacuum vector $\psi$. Since the only representations $\lambda$ of $U(2 N)$ which have non-zero multiplicity satisfy $\lambda_{i}+\lambda_{-i}=1$, the action of the operators $s_{i j}$ with $i \neq-j$ gives zero. Thus a basis in $V_{+}$is given by the vectors

$$
\psi(\lambda)=\frac{1}{C_{\lambda}} s_{1,-1}^{p_{1}} s_{2,-2}^{p_{2}} \ldots s_{N .-N}^{p_{N}} \psi_{0},
$$


where the non-negative integers $p_{i}$ are related to the highest weight by $\lambda_{i}=p_{i}+1$ $=-\lambda_{-i}+1$ for $i=1,2, \ldots, N$. The $C_{\lambda}$ 's are normalization constants. The vectors $\psi(\lambda)$ corresponding to different $\lambda$ 's are orthogonal to each other since they are eigenvectors of the hermitian generators $e_{i i}$. From [M4] we obtain the following formula for the operators $s_{i,-i}$ :

$$
\begin{aligned}
S_{i,-i}= & \sum_{k, l=0}^{i-1} \sum_{\mu_{k}>\mu_{k-1}>\ldots>\mu_{1}=1}^{i} \sum_{v_{l}>v_{l-1}>\ldots>v_{1}=-i}^{-1} e_{i, \mu_{k}} e_{\mu_{k}, \mu_{k-1}} \ldots e_{\mu_{1}, v_{l}} \\
& \left.\times e_{v_{l}, v_{l}-1} \ldots e_{v_{2}, v_{1} v_{v_{1}},-i} e_{\prod_{j=1}}^{k} \frac{1}{t_{i \mu_{j}}-1}\right) \\
& \times\left(\prod_{j=1}^{l} \frac{1}{t_{-i, v_{J}}}\right)\left(\prod_{j=1}^{i-1}\left(t_{i j}-1\right)\right)\left(\prod_{j=-i+1}^{-1} t_{-i, j}\right),
\end{aligned}
$$

where $t_{i j}=e_{i i}-e_{j j}+i-j-1$. Note that because of cancellations the operator $s_{i,-i}$ is in fact a polynomial in the elements $t_{m j}$.

Let $\theta$ be the antilinear antiautomorphism of $\mathscr{U}(\mathrm{gl}(2 N)+\mathbf{a}(N))$ defined by $\theta\left(e_{i j}\right)=e_{j i}$ and $\theta\left(f_{i j}\right)=f_{j i}$. The inner product in $V$ satisfies

$$
\left\langle u \psi_{0}, v \psi_{0}\right\rangle=\left\langle\psi_{0}, \theta(u) v \psi_{0}\right\rangle
$$

(by unitarity relations for the subgroup $U(2 N) \ltimes U A(N) \subset G L(2 N)) \ltimes A(N)$. This allows in principle a determination of the normalization coefficients $C_{\lambda}$. However, things get very complicated pretty soon by increasing the exponents $p_{i}$ and we do not have a closed formula, valid for all $\lambda$ 's.

\section{Highest Weight Representations of the Extension $\widehat{G L}_{2}$}

Let $\Gamma$ denote the space of all smooth sections of the dual determinant bundle $\mathrm{DET}_{2}^{*}$. By definition, a section of $\mathrm{DET}_{2}^{*}$ is a smooth function $\psi: \mathrm{St}_{2} \rightarrow \mathbb{C}$ such that

$$
\psi(w t)=\psi(w) \omega\left(w_{+}, t\right) \quad t \in G L^{2} .
$$

We shall use the metric constructed in [MR] for the bundle $\operatorname{DET}_{2}$. If $(w, \lambda)$ represent a point in $\mathrm{DET}_{2}$, then the length of $(w, \lambda)$ is equal to $|\lambda| l(w)$, where

$$
\begin{gathered}
l(w)=\sqrt{\operatorname{det}_{2}\left(\left|w_{-}\right|^{2}+\left|w_{+}\right|^{2}\right)}, \\
\times \exp \left(-\operatorname{Retr}\left(w_{+}-\frac{1}{2}\left|w_{-}\right|^{2}-\frac{1}{2}\left|w_{+}\right|^{2}-\frac{1}{2}+\frac{1}{8}\left|F_{21}\right|^{2}\right)\right) .
\end{gathered}
$$

(This is not quite the formula given in [MR] because a different cocycle $\omega$ was used there.) We have to check three things:

1) The hermitian part of the operator in the exponent is really a trace class operator: We can write $w_{+}=1+A$, where $A \in I_{2}$. Then

$$
-\frac{1}{2}\left|w_{+}\right|^{2}+\frac{1}{2}\left(w_{+}+w_{+}^{*}\right)-\frac{1}{2}=-\frac{1}{2} A^{*} A,
$$

which is in $I_{1}$. Thus we have to show only that $\frac{1}{4}\left|F_{21}\right|^{2}-\left|w_{-}\right|^{2}$ is in $I_{1}$. In [MR] it was shown that $\frac{1}{2} F_{21}-w_{-}=w_{-} B$, where $B \in I_{2}$. Thus

$$
\left|w_{-}\right|^{2}=\left|\frac{1}{2} F_{21}-w_{-} B\right|^{2}=\frac{1}{4}\left|F_{21}\right|^{2}+\text { operators in } I_{1},
$$

since $F_{21}$ and $w_{-}$are in $I_{4}$. This completes the proof of 1 ). 
2) We have to show that $|(w, \lambda)|=\left|\left(w t, \lambda \omega_{2}\left(w_{+}, t\right)^{-1}\right)\right|$ for $t \in G L^{2}$. But this is equivalent to

$$
\frac{l(w t)}{l(w)}=\left|\omega_{2}\left(w_{+}, t\right)\right|
$$

which can be checked through a simple computation.

3) The function $l$ is strictly positive; this is obvious from the definition.

For each $N \in \mathbb{N}$ we shall consider $G L(2 N)$ as a subgroup of $G L_{2}$ by extending $A \in G L(2 N)$ to an infinite matrix such that $A_{i j}-\delta_{i j}=0$ for $|i|>N$ and $|j|>N$. Since the Grassmannian $\operatorname{Gr}(N)$ is the quotient of $G L(2 N)$ by the subgroup of triangular matrices $\left(\begin{array}{ll}a & b \\ 0 & d\end{array}\right)$, the embedding $G L(2 N) \subset G L_{2}$ defines also an embedding $\operatorname{Gr}(N) \subset \mathrm{Gr}_{2}$. Similarly the finite-dimensional Stiefel manifold $\operatorname{St}(N)$ can be embedded into the space $\mathrm{St}_{2}$ of admissible basis in $\mathrm{Gr}_{2}$. We denote by $\psi^{(N)}$ the restriction of $\psi \in \Gamma$ to the submanifold $\operatorname{St}(N)$. For each $N$ we can define a positive semi-definite inner product in $\Gamma$ by

$$
\left\langle\psi, \psi^{\prime}\right\rangle_{N}=\int_{\operatorname{Gr}(N)} \psi^{(N)} \overline{\psi^{(N)}} l(w)^{-2} d m
$$

where $d m$ is the normalized invariant measure on $\operatorname{Gr}(N)$. The integrand does not depend on the basis $w$ but only on the corresponding point on $\operatorname{Gr}(N)$; this is a consequence of (3.3). The formula (3.4) defines a positive definite inner product in the space of sections of the determinant bundle over $\operatorname{Gr}(N)$. Since the definition of a section over $\operatorname{Gr}(N)$ differs from the the description in (2.5) this point needs some clarification.

In the finite-dimensional case we can write

$$
\omega_{2}\left(w_{+}, t\right)=\operatorname{det} t \frac{\beta\left(w_{+} t\right)}{\beta\left(w_{+}\right)}, \beta\left(w_{+}\right)-e^{-\operatorname{tr} w_{+}} .
$$

To a section $\psi^{(N)}$ over $\operatorname{Gr}(N)$ we can associate a function $\phi: U(2 N) \rightarrow \mathbb{C}$ by

$$
\phi\left(\begin{array}{ll}
a & b \\
c & d
\end{array}\right)=\psi^{(N)}(w) \beta\left(w_{+}\right)^{-1} e^{\frac{1}{8} \operatorname{tr}\left|F_{21}\right|^{2}-\frac{1}{2}},
$$

where $w_{+}=a, w_{-}=c$, and $F_{21}=2 w_{-} w_{+}^{*}=2 c a^{*}$. Then

$$
\phi(g t)=\phi(g) \operatorname{det} t, \text { for } t=\left(\begin{array}{ll}
r & 0 \\
0 & s
\end{array}\right) \in U(2 N),
$$

and therefore $\phi$ is a section in the sense of (2.5). Furthermore, if $\phi^{\prime}$ corresponds to $\psi^{\prime(N)}$, then

$$
\left\langle\psi^{(N)}, \psi^{(N)}\right\rangle_{N}=\int_{\operatorname{Gr}(N)} \phi \overline{\phi^{\prime}} d m .
$$

The formula above follows directly from (3.2) by observing that $\left|w_{+}\right|^{2}+\left|w_{-}\right|^{2}=1$ in the unitary case.

We shall study the action of an abelian extension $\hat{U}_{2}$ of the unitary subgroup $U_{2}$ of $G L_{2}$ in $\Gamma$. Let $(g, q, \lambda)$ be a triple such that $g \in U_{2}, q \in G L^{2} \cap U\left(H_{+}\right)=U^{2}$ and $\lambda$ is a smooth function on $\mathrm{Gr}_{2}$ satisfying

$$
|\lambda(F)|=\frac{l(w)}{l\left(g w q^{-1}\right)}|\alpha(g, q ; w)|^{-1}
$$


where $w$ is any admissible basis at $F \in \mathrm{Gr}_{2},[\mathrm{MR}]$. The action on $\psi \in \Gamma$ is defined by

$$
\begin{gathered}
(T(g, q, \lambda) \psi)(w)=\psi\left(g^{-1} w q\right) \lambda\left(g^{-1} \cdot F\right) \alpha\left(g, q ; g^{-1} w q\right)^{-1}, \\
\alpha(g, q ; w)=e^{-\operatorname{tr}\left[\left(1-q^{-1} a\right)(w+-1)+q^{-1} b\left(\frac{1}{2} F_{21}-w_{-}\right)\right]} .
\end{gathered}
$$

The elements $\left(1, q,\left(\operatorname{det}_{2} q^{-1}\right)^{-1}\right)$, where $q \in U^{2}$ act trivially in $\Gamma$ and the group $\widehat{U}_{2}$ is the quotient of the group of all triples $(g, q, \lambda)$ modulo the subgroup consisting of the elements $\left(1, q,\left(\operatorname{det}_{2} q^{-1}\right)^{-1}\right)$. Since already the smaller group consisting of the triples $(g, q, \lambda)$ with

$$
\frac{\lambda}{|\lambda|}=e^{i \eta+\operatorname{tr} \xi(F-\varepsilon)}, \quad-\xi^{*}=\xi \in\left(\begin{array}{cc}
I_{2} & I_{4 / 3} \\
I_{4 / 3} & I_{2}
\end{array}\right), \quad \eta \in \mathbb{R}
$$

acts in $\Gamma$ we shall restrict the definition of $\hat{U}_{2}$ to these elements. The condition (3.9) guarantees that the action of $\hat{U}_{2}$ preserves the metric, i.e.

$$
\left|\left(g w q^{-1}, \alpha(g, q ; w) \lambda(F)\right)\right|=|(w, 1)| .
$$

If we had an $U_{2}$ invariant measure $d m$ on the infinite-dimensional Grassmannian $\mathrm{Gr}_{2}$, then (3.11) would imply that the following inner product in $I$ would be invariant under the action of $\hat{U}_{2}$ :

$$
\left\langle\psi, \psi^{\prime}\right\rangle=\int_{\mathrm{Gi}_{2}} \psi \bar{\psi}^{\prime} l(w)^{-2} d m .
$$

Since such a measure is not known (in contrast to the case of $\mathrm{Gr}_{1}$, see [Pi]) we shall use only the topology determined by the family $\langle\cdot, \cdot\rangle_{N}$ of semi-definite inner products; the inner product $\langle\cdot, \cdot\rangle_{N}$ is invariant under those elements of $\hat{U}_{2}$ which are represented by triples $(g, q, \lambda)$ such that $g \in U(2 N)$.

As a vector space the Lie algebra $\hat{\mathbf{u}}_{2}$ of $U_{2}$ is a direct sum of the vector space $\mathbf{u}_{2}$ and the vector space consisting of the pairs $(\eta, \xi)$ above. We shall extend the representation of $\hat{\mathbf{u}}_{2}$ in $\Gamma$ (corresponding to the group representation $T$ ) by complex linearity to a representation of the complex Lie algebra $\hat{\mathrm{g}} l_{2}$; this latter algebra is a direct sum of the vector spaces $\mathrm{gl}_{2}$ and the space consisting of the $(\eta, \xi)$ 's with $\eta \in \mathbb{C}$ and $\xi$ an arbitrary complex matrix such that the diagonal blocks are in $I_{2}$ and the off-diagonal blocks in $I_{4 / 3}$. The commutation relations are given by $(1.5)$. In the Weyl basis $\left(e_{i j}, f_{i j}\right)$ we have

$$
\left[e_{i j}, e_{k l}\right]=\delta_{j k} e_{i l}-\delta_{i l} e_{k j}+c_{2}\left(e_{i j}, e_{k l}\right),
$$

where the cocycle $c_{2}$ is defined by

$$
c_{2}\left(e_{i j}, e_{k l}\right)= \begin{cases}\frac{1}{2} \delta_{j k} f_{i l}-\frac{1}{2} \delta_{i l} f_{k j}+\delta_{j k} \delta_{i l}, & i, l>0>k, j \\ \frac{1}{2} \delta_{j k} f_{i l}-\frac{1}{2} \delta_{i l} f_{k j}-\delta_{j k} \delta_{i l}, & k, j>0>i, l \\ 0, & \text { otherwise }\end{cases}
$$

The functions $f_{i j}$ commute among themselves but

$$
\left[e_{i j}, f_{k l}\right]=\delta_{j k} f_{i l}-\delta_{i l} f_{k j}
$$

The hermiticity relations following from the unitarity properties of the group action are

$$
\left\langle\psi, e_{i j} \psi^{\prime}\right\rangle_{N}=\left\langle e_{j i} \psi, \psi^{\prime}\right\rangle_{N} \quad \text { for } \quad|i|,|j| \leqq N
$$


and $f_{j i}$ is the hermitian conjugate of $f_{i j}$ with respect to any of the inner products $\langle\cdot, \cdot\rangle_{N}$.

As in the finite-dimensional case we define the Cartan subalgebra $\mathbf{h} C \mathrm{gl}_{2}$ spanned by the generators $e_{i i}, 0 \neq i \in \mathbb{Z}$ and the "triangular" subalgebra $\mathbf{k}_{+}$spanned by the vectors $e_{i,-j}$ with $i, j>0$ and by $e_{i j}, e_{-j,-i}$ with $0<i<j$. The subalgebra $\mathbf{k}_{-}$is the complement of $\mathbf{h} \oplus \mathbf{k}_{+}$in $\mathrm{gl}_{2}$. This is not the standard splitting; the latter is defined by the basis $e_{i,-j}$ with $i, j>0$ and $e_{j i}, e_{-i,-j}$ with $0<i<j$ of the subalgebra $\mathbf{k}_{+}$. These two splittings are not connected by any inner automorphism.

According to $[\mathrm{MR}]$ the space $\Gamma$ contains a (unique up to a multiplicative constant) vacuum vector $\psi_{0}$ which is characterized by the property that

$$
e_{i j} \psi_{0}=e_{-i,-j} \psi_{0}=e_{i,-j} \psi_{0}=0
$$

for all $i, j>0$. In particular, $\mathbf{k}_{+} \psi_{0}=0$. Explicitly, $\psi_{0}(w)=\mathrm{det}_{2} w_{+}$, which shows that $\psi_{0}$ is in fact a holomorphic section of DET*. Define a set of new elements in $\mathrm{gl}_{2}$ by

$$
e_{i j}^{\prime}= \begin{cases}e_{i j}-\frac{1}{2} f_{i j}, & i<0 \leqq j \\ e_{i i}-1, & i=j<0 \\ e_{i j}, & \text { otherwise. }\end{cases}
$$

From (3.13) and (3.14) we get

$$
\left[e_{i j}^{\prime}, e_{k l}^{\prime}\right]=\delta_{j k} e_{i l}^{\prime}-\delta_{i l} e_{k j}^{\prime}
$$

Thus the elements $e_{i j}^{\prime}$ generate the Lie algebra gl $(\infty)$ consisting of all finite linear combinations of the primed vectors. We define the operators $s_{i j}^{\prime}$ in the enveloping algebra of $\mathrm{gl}_{2}$ as in the finite-dimensional case except that the generators $e_{i j}$ are replaced by the generators $e_{i j}^{\prime}$. From the commutation relations (3.18) if follows that the modified operators $s_{i j}^{\prime}$ satisfy the relations (2.6). (Note that the $e_{i j}$ 's satisfy the same commutation relations with the $f_{k l}$ 's as the generators $e_{i j}^{\prime}$.) Thus the following vectors are annihilated by $\mathbf{k}_{+}=\mathbf{k}_{+}^{\prime}$ :

$$
\psi(\lambda)=s_{1,-1}^{p_{1}-1} s_{2,-2}^{\prime p_{2}} \ldots s_{N,-N}^{p_{N}} \psi_{0},
$$

where the weight $\lambda \in \mathbf{h}^{*}$ is given by $\lambda_{i}=p_{i}=-\lambda_{-i}$ for $1 \leqq i \leqq N$ and $\lambda_{i}=0$ for $|i|>N$; $\lambda_{i}$ is the eigenvalue of $e_{i i}$.

Let us define the $\hat{\mathrm{g}} \mathrm{l}_{2}$ invariant subspace of algebraic sections $\Gamma_{\mathrm{alg}}=\mathscr{U}\left(\hat{\mathrm{g}} \mathrm{l}_{2}\right) \psi_{0} \subset \Gamma$. Denote by $\mathbf{k}_{-}^{\prime}$ the Lie algebra spanned by the vectors $e_{i,}^{\prime}$ with $e_{i j} \in \mathbf{k}_{-}$. The following proposition gives a decomposition of $\Gamma$ into representations of the Lie algebra $\mathrm{gl}(\infty)$. Note that the vectors $\psi(\lambda)$ are not extremal vectors in a highest weight representation of $\mathrm{gl}(\infty)$ in the normal sense since our $\mathbf{k}_{+}^{\prime}$ does not define a triangular splitting equivalent to the standard splitting to upper and lower triangular matrices in $\mathrm{gl}(\infty)$.

Theorem 3.19. The direct sum $V$ of the subspaces $\mathscr{U}\left(\mathbf{k}_{-}^{\prime}\right) \psi(\lambda)$ is dense in $\Gamma_{\text {alg }}$ with respect to the topology defined by the seminorms $\|\psi\|_{N}=\sqrt{\langle\psi, \psi\rangle_{N}}$. Here the weight $\lambda$ runs through all the values such that $\lambda_{i}=-\lambda_{-i}, \lambda_{1} \geqq \lambda_{2} \geqq \ldots$ and $\lambda_{i}=0$ when $|i| \gg 0$.

Proof. Let $\phi \in \Gamma_{\text {alg }}$. The mapping $\psi \mapsto \psi^{(N)}$ maps $\Gamma_{\text {alg }}$ surjectively to the space of algebraic sections over the finite-dimensional submanifold $\operatorname{Gr}(N)$, that is, onto the space of sections $\mathscr{U}(\hat{\mathrm{g}} l(2 N)) \psi_{0}^{(N)}$. In the case of $\mathrm{gl}(2 N)$ we know that the space 
$\mathscr{U}(\hat{\mathrm{g} l}(2 N)) \psi_{0}$ decomposes to a sum of subspaces of the type $\mathscr{U}\left(\mathbf{k}_{-}\right) \psi(\lambda)$. It follows that there is an element $\phi_{N} \in V$ such that the restriction $\phi_{N}^{(N)}$ is equal to $\phi^{(N)}$. But this means that

$$
\left\|\phi-\phi_{N}\right\|_{M}=0, \text { for } M \leqq N
$$

so that $\lim _{N \rightarrow \infty}\left\|\phi-\phi_{N}\right\|_{M}=0$ for all $M$.

Suppose that the basis vectors $e_{i}$ in $L_{2}(S)$ are eigenvectors of the Dirac Hamiltonian $\mathscr{H}=\sum \alpha_{k} D_{k}(A)+\gamma_{0} m$ on a 3 -dimensional spin manifold; $A$ is a vector potential corresponding to a compact gauge group $G$ acting on the internal symmetry indices of the Dirac spinor. Let $\varepsilon_{i}$ be the eigenvalue of $\mathscr{H}$ associated to $e_{i}$. We assume that the basis is labelled in such a way that $\ldots \varepsilon_{2} \leqq \varepsilon_{1}<0 \leqq \varepsilon_{-1} \leqq \varepsilon_{-2} \ldots$ The second quantized Hamiltonian acting in $\Gamma$ is then

$$
\mathscr{H}=\sum \varepsilon_{i} e_{i i} .
$$

The vacuum $\psi_{0}$ is an eigenvector with eigenvalue 0 . However, the representation theory of $\widehat{G L}$ differs from the representation theory of linear groups associated to gauge groups in $1+1$ dimensional quantum field theory in an essential way: The highest weight vector $\psi_{0}$ is not a vector of lowest energy! The second quantized Hamiltonian is not even bounded below! This property is not restricted to the representation in $\Gamma$ but it is much more general; we shall here give a proof for any unitary Hilbert space representation, but one can generalize this to the case of a denumerable family of semi-definite inner products under suitable conditions on the seminorms.

Proposition 3.21. Let a hermitian representation of $\hat{g} l_{2}$ be given in a vector space $V$ with positive definite inner product. Then there is no vector $0 \neq v \in V$ of lowest energy.

Proof. Assume that $v$ is an eigenvector of $\mathscr{H}$ corresponding to the smallest eigenvalue $\mu$. If $i \geqq 0>j$, then $f_{i j} v$ is an eigenvector to the eigenvalue $\mu+\varepsilon_{i}-\varepsilon_{j}<\mu$, and therefore $f_{i j} v=0$. Similarly, $e_{i j} v=0$ for $i \geqq 0>j$. Now

$$
\left\|f_{j i} v\right\|^{2}=\left\langle f_{j i} v, f_{j i} v\right\rangle=\left\langle v, f_{i j} f_{j i} v\right\rangle=0,
$$

since the $f_{i j}$ 's commute among themselves. Thus also $f_{j i} v=0$ for $i \geqq 0>j$. Furthermore,

$$
f_{k l} v=\left[e_{k j}, f_{j l}\right] v=0
$$

when $k \neq l \geqq 0$ and $j<0$. Similarly,

$$
f_{k l} v=-\left[e_{j l}, f_{k j}\right] v=0
$$

for $k \neq l<0$ and $j>0$. Since $f_{i i}-f_{j j}=\left[e_{i j}, f_{j i}\right]$ we have $\left(f_{i i}-f_{j j}\right) v=0$ for $i \geqq 0>j$. Since $\sum \alpha_{i} e_{i i}$ is in $\hat{\mathrm{g}} l_{2}$ for any bounded sequence of numbers $\alpha_{i}$, we know that

for such a sequence.

$$
\sum \alpha_{i}\left\langle v, e_{i i} v\right\rangle \text { converges }
$$

Let $\alpha_{1}, \alpha_{2}, \ldots$ be a sequence of complex numbers such that $\left|\alpha_{1}\right|^{4}$ $+\left|\alpha_{2}\right|^{4}+\ldots<\infty$. Now $\sum_{i} \alpha_{i} e_{-i, i}$ is in $\hat{g} l_{2}$. But

$$
\left\|\sum_{i} \alpha_{i} e_{-i, i} v\right\|^{2}=\sum\left|\alpha_{i}\right|^{2}\left\langle v,\left(e_{i i}-e_{-i,-i}\right) v\right\rangle+\sum\left|\alpha_{i}\right|^{2},
$$


where we have used the fact that $\left(f_{i i}-f_{-i,-i}\right) v=0$. The first term on the right in (3.23) converges by (3.22) but the second term diverges for a typical element in $I_{4}$, a contradiction.

\section{The Second Quantized Coupled Dirac-Yang-Mills System}

In a previous article [M2] we described a geometric formulation of string theory when the background space is a compact group manifold and especially the role of the homogeneous space Diff $S^{1} / S^{1}$ and its holomorphic structure. Here we want to show how the same method can be used to describe the anomaly cancellation for the second quantized Dirac-Yang-Mills system. Let us quickly recapitulate the main idea of [M2]. We consider first a classical system whose phase space is the group $L G$ of smooth loops in $G$. The dynamics on $L G$ is defined by the classical Hamiltonian

$$
\int_{S^{1}}\left\langle f(\phi)^{-1} f^{\prime}(\phi), f(\phi)^{-1} f^{\prime}(\phi)>d \phi,\right.
$$

where $\langle$,$\rangle is the Killing form of G$ and $f \in L G$. The symplectic form on $L G$ is

$$
(X, Y)=\text { const } \times \int_{S^{1}}\left\langle X(\phi), Y^{\prime}(\phi)\right\rangle d \phi,
$$

where the tangent vectors on $L G$ are thought of as maps from $S^{1}$ to the Lie algebra of $G$. The system is quantized by constructing a complex line bundle $E$ with a connection such that the curvature is equal to the symplectic form. The quantum Hamiltonian $H$ is the covariant Laplacian acting on sections of the line bundle $E$. The operator $H$ does not commute with the group Diff $S^{1}$ which acts on the holomorphic sections of the bundle $E$ through the Sugawara construction. Equivalently, the group Diff $S^{1}$ does not leave invariant the metric and the normal ordering prescription which are used to define the quantum Hamiltonian; only the subgroup $S^{1}$ commutes with $H$. Thus one is forced to consider a whole bundle $B$ of quantum systems parametrized by the manifold $M=\operatorname{Diff} S^{1} / S^{1}$. The fiber of the bundle $B$ is the space of holomorphic sections of the bundle $E$. Strictly speaking, the fiber is different at different points on the base space $M$, but the base is topologically trivial, and therefore the bundle can be trivialized, that is, the different fibers can be identified in a continuous manner. The group Diff $S^{1}$ acts in the bundle through the natural action on the base space and the Sugawara construction in the fiber. Because of the central term in the Virasoro commutation relations there is no invariant vacuum vector. This is reflected also as the nonvanishing of the curvature of the bundle $B$. The curvature can be canceled by tensoring $B$ with a canonical holomorphic line bundle over $M$. The central term of the Virasoro algebra acting on the sections of the new bundle vanishes and there is a Diff $S^{1}$ invariant vacuum.

Let $M$ be a compact three dimensional spin manifold and $D_{0}$ the Dirac operator on $M$. We assume also that there is compact gauge group $G$ acting on the Dirac spinors, and we denote by $A$ an associated vector potential which takes values in the Lie algebra of $G$ (for simplicity we assume that the vector bundle where $G$ lives is trivial). We denote by $\mathscr{A}$ the space of all smooth vector potentials 
$A$. Let $D_{A}$ denote the Dirac operator corresponding to the vector potential $A$. We write the space $H$ of square integrable Dirac spinors as a direct sum $H=H_{+} \oplus H_{-}$ of positive and negative energy subspaces with respect to the free Dirac operator $D_{0}$. We can define a similar decomposition $H=H_{+}(A) \oplus H_{-}(A)$ for each smooth vector potential $A$. One can show slightly modifying the proof in [MR, Sect. 2], that the difference $D_{0}-D_{A}$ has off-diagonal blocks, with respect to the decomposition $H=H_{+} \oplus H_{-}$, belonging to the Schatten ideal $I_{4}$. From this follows easily that the projection of $H_{+}(A)$ to $H_{-}$is in $I_{4}$ and the projection $H_{+}(A) \rightarrow H_{+}$is a Fredholm operator. This means that the plane $H_{+}(A)$ is an element of the Grassmannian $\mathrm{Gr}_{2}$ defined by the decomposition $H=H_{+} \oplus H_{-}$. The mapping $A \mapsto H_{+}(A)$ is not quite continuous; it has a discontinuity at those points $A$ where one of the eigenvalues of the Dirac operator $D_{A}$ crosses the zero level. However, this problem can be treated by the following observation, [Se]: Let $\alpha$ be any real number, $A$ a smooth vector potential and $\widetilde{F}_{A}(\alpha)$ the Fock space formed with respect to the splitting of the spectrum of $D_{A}$ to the half-lines $[\alpha, \infty[$ and $]-\infty, \alpha[$. Then $\widetilde{F}_{A}(\alpha)$ and $\widetilde{F}_{A}(\beta)$ are canonically isomorphic up to a multiplicative phase factor.

Each point $W \in \mathrm{Gr}_{2}$ defines a fermionic vector space consisting of sections $\Gamma(W)$ of the bundle DET ${ }_{2}^{*}(W)$; let $B$ be the vector bundle with base $\mathrm{Gr}_{2}$ fiber $\Gamma(W)$ at $W \in \mathrm{Gr}_{2}$. The pull-back of the bundle $B$ with respect to the mapping $A \mapsto H_{+}(A)$ from $\mathscr{A}$ to $\mathrm{Gr}_{2}$ is the vector bundle needed for describing the interacting DiracYang-Mills system. However, it is more convenient to work with the bundle $B$ than with the pull-back because of the complications due to discontinuities mentioned above (strictly speaking, the pull-back exists only as a bundle of projective fermionic vector spaces).

Fix a point $W \in \mathrm{Gr}_{2}$. As before we view sections $\psi \in \Gamma(W)$ as equivariant functions $\psi: \mathrm{St}_{2} \rightarrow \mathbb{C}$,

$$
\psi(w t)=\psi(w) \omega_{2}(w, t) .
$$

Let $\left\{e_{i}\right\}_{l}>0$ be a fixed basis in $H_{+}$and define the matrix $w_{+}$for each admissible basis $w$ with respect to $e$ as explained in the introduction. The matrix $w_{+}$is of the type $1+$ a Hilbert-Schmidt operator. The reader might object that we have now defined the space of sections at the point $W$ with respect to the reference frame at $H_{+}$and not with respect to a frame at $W$. This does not really make any difference for the following reason: If $\left\{e_{i}^{\prime}\right\}$ is an admissible basis of $W$ (with respect to the basis $\left\{e_{i}\right\}$ ), then a basis $\left\{w_{i}\right\}$ of any Grassmannian plane is admissible with respect to $\left\{e_{i}^{\prime}\right\}$ if and only if it is admissible with respect to $\left\{e_{i}\right\}$. The bundle $B$ is topologically trivial, $B \cong B_{H_{+}} \times \mathrm{Gr}_{2}$. However, the vacuum sector of $B$ is non-trivial. The vacuum vector in $B_{H_{+}}$is the holomorphic section of Det* which can be characterized by either of the following conditions:

(1) It is an eigenvector of $\hat{\mathscr{H}}$ corresponding to the (lowest) eigenvalue 0 ,

(2) it is projectively invariant under a certain triangular subgroup $N$ of $\widehat{G L}_{2}$.

The subgroup $N$ is a homomorphic image of the subgroup of $G L_{2}$ consisting of the block triangular matrices $\left(\begin{array}{ll}a & 0 \\ c & d\end{array}\right)$, where the blocks of $g$ are defined with respect to the decomposition $H=H_{+} \oplus H_{-}$. The vacuum at $W=H_{+}$is explicitly given by the formula

$$
\psi_{H+}(w)=\operatorname{det}_{2} w_{+}
$$


We define now the vacuum vector at $W=g H_{+}$to be the section $\psi_{W}$ (unique, up to a constant) which is projectively invariant under the group $N_{W}$ which is defined similarly as $N$ but using the splitting $L_{2}(S)=W \oplus W^{\perp}$. In other words, the vacuum at $W=g H_{+}$(where $g$ can be chosen to be unitary) is the lowest energy section for the quantization of the Hamiltonian $g D_{0} g^{-1}$. Note that the vacuum is the same for all g's which differ from each other by a left multiplication by a unitary operator of the type $\left(\begin{array}{ll}a & 0 \\ 0 & d\end{array}\right)$ so that the vacuum really depends only on the base point $W$ and not of the operator $g$.

Let Vac denote the vacuum bundle; by definition, Vac is the complex line bundle over $\mathrm{Gr}_{2}$ such that the fiber at $W \in \mathrm{Gr}_{2}$ is the complex line in $B_{W}$ spanned by the vacuum vector.

The subbundle $\operatorname{Vac} \subset B$ is not invariant under the action of $\widehat{G L}_{2}$. Namely, let $W \in \mathrm{Gr}_{2}$ be arbitrary and $\psi \in \mathrm{Vac}_{W}$. The action of an element of $\widehat{G L}_{2}$ on a section of $\mathrm{DET}_{2}^{*}$ is given by the formula $\psi \mapsto \psi^{\prime}$,

$$
\psi^{\prime}(w)=\mu\left(g^{-1} \cdot F\right)^{-1} \alpha\left(g, q ; g^{-1} w q\right)^{-1} \psi\left(g^{-1} w q\right),
$$

where $g=\left(\begin{array}{ll}a & b \\ c & d\end{array}\right), \mu$ is an arbitrary function on $\mathrm{Gr}_{2}, q$ is a linear operator such that $a q^{-1}-1$ is in $I_{2}$ and $\alpha$ is the cocycle defined in (3.10). The section $\psi^{\prime}$ is not even holomorphic because of the non-holomorphic factor $\alpha$. The situation differs in this respect from the $1+1$ dimensional case. In that case one can put $\alpha \equiv 0$ and the relevant (central) extension of $G L_{1}$ acts in Vac. However, there also Vac is twisted and there does not exist any non-zero section invariant under $\widehat{G L}_{1}$.

In order to define an invariant vacuum we shall proceed as in the string theoretic example. We shall define a new bundle $\bar{B}$ as the tensor product of the Fock bundle $B$ with the line bundle Det $_{2}$. The sections of the bundle $\bar{B}$ can be considered as maps $\psi: \mathrm{St}_{2} \times \mathrm{St}_{2} \rightarrow \mathbb{C}$ such that

$$
\psi(w s, f t)=\psi(w, f) \omega\left(w_{+}, s\right) \omega\left(f_{+}, t^{-1}\right)
$$

for all $w, f \in \mathrm{St}_{2}$ and $s, t \in G L^{2}$. For each fixed $f \in \mathrm{St}_{2}$ the function $w \mapsto \psi(w, f)$ gives an element in the fiber $B_{W}$ over the base point $W=\operatorname{pr}(w) \in \mathrm{Gr}_{2}$. In order to make the structure of the bundle $\bar{B}$ a bit more transparent we shall redefine the cocycle in (4.6) using the mapping $\psi \mapsto \psi^{\prime}, \psi^{\prime}(w, f)=\psi(w, f) \exp \left(\operatorname{tr}\left(f_{+}^{e}-w_{+}^{f}-1+w_{+}^{e}\right)\right)$, where for any $w, f \in \mathrm{St}_{2} w_{+}^{f}$ denotes the matrix defined by

$$
w_{i}=\sum_{j}\left(w_{+}^{f}\right)_{j i} f_{j} \text {. }
$$

In particular, $w_{+}^{e}$ is the same as $w_{+}$. The primed functions satisfy the conditions

$$
\psi^{\prime}(w t, f)=\psi^{\prime}(w, f) \omega\left(w_{+}^{f}, t\right), \psi^{\prime}(w, f t)=\psi^{\prime}(w, f) \omega\left(w_{+}^{f}, t^{-1}\right) .
$$

Here the cocycle defining the space $B_{W}$ at $W=p r(f)$ is fixed "relative to the frame of reference" $f$. For different frames $f$ at the same base point $W$ the spaces are isomorphic but not naturally isomorphic. Note that $\psi^{\prime}$ is invariant under simultaneous right multiplication of $w$ and $f$ by an element $t \in G L^{2}$. After this redefinition of the wave functions we shall drop the primes. 
In the bundle $\bar{B}$ the vacuum line bundle is trivial. A global everywhere nonvanishing section is

$$
\psi_{0}(w, f)=\operatorname{det}_{2} w_{+}^{f} .
$$

Of course, $\psi_{0}$ has zeros as a function of the two variables $w$ and $f$, but as a section of $\bar{B}$ it is everywhere non-vanishing since at no point $f$ the map $w \mapsto \psi_{0}(w, f)$ is the zero function. An element $g=\left(\begin{array}{ll}a & b \\ c & d\end{array}\right) \in G L_{2}$ acts on the sections of the bundle $\bar{B}$ by

$$
(g \cdot \psi)(w, f)=\psi\left(g^{-1} w q, g^{-1} f q\right),
$$

where $q$ is any operator such that $a q^{-1}-1 \in I_{2}$. The right-hand-side does not depend on the choice of $q$, since $\psi(w t, f t)=\psi(w, f)$ for $t \in G L^{2}$. The matrix $w_{+}^{f}$ is invariant under simultaneous action of $(g, q)$ on $w, f$, and therefore $g \psi_{0}=\psi_{0}$ for all $g \in G L_{2}$.

As we have remarked, the space of holomorphic sections of DET D $_{2}^{*}$ is not invariant under the action of the group $\widehat{G L}_{2}$. Let us define the Fock bundle $\mathscr{F} \subset B$ as the vector bundle over $\mathrm{Gr}_{2}$ such that the fiber at the point $W$ consists of the holomorphic sections of the bundle DET*. The modified Fock bundle is $\overline{\mathscr{F}}=\mathscr{F} \otimes \mathrm{DET}_{2}$, and it contains as a subbundle the vacuum line bundle Vac. The sections of the bundle $\overline{\mathscr{F}}$ form the space of states for the quantized Dirac-YangMills system.

For each sequence $(i)=\left(i_{1}, i_{2}, i_{3}, \ldots\right)$ of integers such that the sets $\mathbb{N} \backslash(i)$ and $-\mathbb{N} \cap(i)$ are finite we define a holomorphic section of $\mathrm{DET}_{2}^{*}$ by the formula

$$
\psi_{(i)}(w)=\operatorname{det}_{2} w(i) \cdot e^{\operatorname{tr}(w(i)-w+)},
$$

where $w(i)$ is the matrix obtained from the $\mathbb{Z} \times \mathbb{N}$ matrix $\left(\begin{array}{c}w_{+} \\ w_{-}\end{array}\right)$by selecting the rows labelled by the integers $(i)$. In particular, when $(i)=\mathbb{N}$, we get $\psi_{(i)}(w)=\operatorname{det}_{2} w_{+}$. We define an inner product in the space of holomorphic sections of DET* by declaring that the vectors $\psi_{(i)}$ form an orthonormal system.

We can now define a fiber metric in $\overline{\mathscr{F}}$ such that the functions $\Psi_{(i)}(w, f)$ $=\operatorname{det}_{2} w^{f}(i)$ form an orthonormal system of sections of $\overline{\mathscr{F}}$. If we had a (quasi)invariant measure $d m$ on the Grassmannian $\mathrm{Gr}_{2}$ we could now define an inner product in the space of square integrable sections by setting

$$
\left\langle\sum \alpha_{(i)} \Psi_{(i)}, \sum \beta_{(j)} \Psi_{(j)}\right\rangle=\int_{\mathrm{Gr}_{2}} \sum \alpha_{(i)} \overline{\beta_{(i)}} d m,
$$

where $\alpha_{(i)}$ and $\beta_{(i)}$ are measurable functions on $\mathrm{Gr}_{2}$. There is a quasi-invariant measure on the Grassmannian $\mathrm{Gr}_{1}$ modelled by Hilbert-Schmidt operators [Pi]; however, it is not clear whether one can generalize the approach of [Pi] to the case of $\mathrm{Gr}_{2}$.

Acknowledgements. The main part of this work was completed at the Arnold Sommerfeld Institute, Technical University of Clausthal, and I wish to thank Prof. H.D. Doebner and other members of the Institute for the pleasant working atmosphere in Clausthal. The research was supported by the von Humboldt Stiftung. I am grateful to D. Pickrell for pointing out errors in the first version of this paper. 


\section{References}

[BR] Bowick, M., Rajeev, S.: Anomalies as curvature in complex geometry. Nucl. Phys. B 296, 1007 (1987)

[C] Connes, A.: Non-commutative differential geometry. Publ. Math. I.H.E.S. 62, 257 (1985)

[F] Faddeev, L.D.: Operator anomaly for the Gauss Law. Phys. Lett. 145 B, 81 (1984)

[H] van den Hombergh, A.: Harish-Chandra modules and representations of step algebras. Ph. D. thesis, Nijmegen (1976)

[Hu] Humphreys, J.E.: Introduction to Lic algebras and representation theory. Berlin, Heidelberg, New York: Springer 1980

[J] Jackiw, R., Johnson, K.: Anomalies of the axial vector current. Phys. Rev. 182, 1459 (1969)

[Kac] Kac, V.: Infinite dimensional Lie algebras. Cambridge: Cambridge University Press 1985

[KP] Kac, V., Peterson, D.H.: Lectures on infinite wedge representations and the MKP hierarchy. Proc. of the Montreal Summer School (1985) on Completely Integrable Systems

[L] Littlewood, D.: The theory of group characters and and matrix representations of groups. Oxford: Oxford University Press 1950

[M1] Mickelsson, J.: Chiral anomalies in even and odd dimensions. Commun. Math. Phys. 97, 361 (1985). Kac-Moody groups, topology of the Dirac determinant bundle and fermionization. Commun. Math. Phys. 110, 173 (1987)

[M2] Mickelsson, J.: String quantization on group manifolds and the holomorphic geometry of Diff $S^{1} / S^{1}$. Commun. Math. Phys. 112, 653 (1987)

[M3] Mickelsson, J.: Step algebras of semi-simple subalgebras of Lie algebras. Rep. Math. Phys. 4, $307(1973)$

[M4] Mickelsson, J.: An explicit basis for the reduction $U(n+m) \downarrow U(n) \times U(m)$. J. Math. Phys. 12, $2378(1971)$

[MR] Mickelsson, J., Rajeev, S.: Current algebras in $d+1$ dimensions and determinant bundles over infinite-dimensional Grassmannians. Commun. Math. Phys. (in press)

[NM] Nagel, J.G., Moshinsky, M.: Operators that lower and raise the irreducible representations of $U_{n-1}$ in an irreducible representation space of $U_{n}$. J. Math. Phys. 6, 682 (1965)

[Pi] Pickrell, D.: Measures in infinite-dimensional Grassmann manifolds. J. Funct. Anal. 70, 323 (1987)

[PS] Pressley, A., Segal, G.: Loop groups and their representations. Oxford: Clarendon Press 1986

[R] de B. Robinson, G.: Representation theory of symmetric group. Edinburg: Edinburgh University Press 1961

[Se] Segal, G.: Unpublished preprint. Oxford (1985)

[S] Singer, I.: Families of Dirac operators with applications to physics. Asterisque 323 (1985)

[Z] Zhelobenko, D.P.: $S$-algebras and Verma modules over reductive Lie algebras. Sov. Math. Dokl. 28, No. 3, 696 (1983)

Communicated by A. Jaffe

Received October 30, 1987; in revised form February 17, 1988 
